\title{
A report on the First Asia-Pacific Gastric Cancer Conference
}

\author{
Ahmad Sudirman, Eric Gan, and Jimmy B.Y. So \\ Department of Surgery, Yong Loo Lin School of Medicine, National University of Singapore, National University Hospital, 5 Lower Kent \\ Ridge Road, Singapore 119074
}

\section{Introduction}

The First Asia-Pacific Gastric Cancer Conference was held on November 20-22, 2006, at the Clinical Research Centre, National University of Singapore (NUS). The aim of this conference was to provide a platform for surgeons, gastroenterologists, oncologists, primary healthcare physicians, and scientists in the region to share their experiences in gastric cancer treatment and research. The conference was very comprehensive: it consisted of four symposia, three hands-on workshops, live demonstrations, plenary lectures, a teleconference, and poster presentations. A total of 206 international delegates from 18 countries participated in this conference, and 26 poster presentations were displayed during the conference.

\section{First day. November 20, 2006}

The conference was officially opened by Jimmy So (Department of Surgery, Yong Loo Lin School of Medicine, NUS, National University Hospital [NUH]), Chairman of the First Asia-Pacific Gastric Cancer Conference, and K. Satkunanathan, Director of Medical Services, Ministry of Health, Singapore. Chuen-Neng Lee, Head and Chief, Department of Surgery, Yong Loo-Lin School of Medicine, NUS, delivered the keynote address, after which the conference began.

Symposium 1. Gastric Cancer: Biology and Genetics (Chaired by K.M. Chu, University of Hong Kong, Hong Kong, and Khay-Guan Yeoh, National University of Singapore, Singapore)

Offprint requests to: J.B.Y. So
Five topics were presented in this symposium. The first part of the morning session was on the epidemiology of gastric cancer, with a presentation from KeeSeng Chia, NUS. He emphasized the decline in the incidence of gastric cancer worldwide and the rising trend of cardial cancer in Western countries. He also highlighted the central role of Helicobacter pylori infection in noncardia gastric cancer and, in contrast, the reduced risks of gastric cardia cancer associated with $H$. pylori seropositivity. Yoshiaki Ito, from the Oncology Research Institute, Singapore, spoke about carcinogenesis and molecular biology. He explained the relationship between $R U N X 3$, a gastric tumor suppressor gene, and the tumorigenicity and intestinalization of gastric epithelial cells [1]. Next, Soo-Chin Lee, an oncologist from the NUH, Singapore, gave a concise review of gastric cancer genetics. She highlighted several hereditary gastric cancer syndromes and the high incidence of stomach cancer in Asian families with hereditary nonpolyposis colon cancer (HNPCC). She recommended that gastric cancer surveillance should be considered for Asian HNPCC kindreds. Finally, she summarized the current available genetic tests for identifying high-risk families for gastric cancer. Enders Ng, from the Chinese University of Hong Kong, discussed the role of $H$. pylori in gastric carcinogenesis. He highlighted a few randomized controlled trial studies regarding the impact of H. pylori eradication on gastric cancer carcinogenesis $[2,3]$. The studies showed that eradication retarded the progression of atrophy and intestinal metaplasia in the stomach, but did not reverse either the intestinal metaplasia or glandular atrophy. The last speaker, Patrick Tan, from the Genome Institute of Singapore, talked about the application of tissue microarrays in gastric cancer research [4]. He discussed patterns of gene expression correlated to gastric cancer regulation that may predict survival. He gave as an example that the expression of the phospholipase A2 Group IIA gene in gastric cancer was associated with prolonged survival. 
Symposium 2. Diagnosis and Treatment of Early Gastric Cancer (Chaired by John Meenan, St. Thomas Hospital, United Kingdom, and Lawrence Ho, NUS, Singapore)

This symposium generated much interest among the members of the audience. Five speakers from tertiary centers throughout the region discussed their experience in the diagnosis and treatment of early gastric cancer. Priyanti Kumarasinghe, a pathologist from the NUS, compared and contrasted the Japanese and Western definitions of early gastric cancer. The incidence of early gastric cancer was noted to be higher in countries where asymptomatic patients are screened (16\%-24\% in the west and $30 \%-50 \%$ in Japan). She also highlighted how the differences in criteria for the diagnosis of adenocarcinoma between the two settings led to major disparities between both the incidence and the outcome of treatment of high-grade dysplasia and early gastric cancer. Then Kenjiro Yasuda, from the Kyoto Second Red Cross Hospital, Japan, described current and state-of-the-art endoscopic techniques in the diagnosis of early gastric cancer. Philip Chiu, from the Chinese University of Hong Kong, discussed advances in endoscopic imaging technology which can potentially enhance the detection and recognition of early gastric cancer. These promising new technologies, such as narrow band imaging, autofluorescence imaging, and confocal endoscopy, may transform the management of early gastric cancer to a one-stage endoscopic procedure [5]. Ichiro Oda, from National Cancer Center Hospital, Japan, described current techniques for endoscopic mucosal resection (EMR) in the treatment of early gastric cancer, encompassing the indications and results of EMR [6]. Lastly, Enders Ng presented his center's experience with endoscopic submucosal dissection (ESD).

Plenary lecture 1. Adenocarcinoma of Gastroesophageal Junction (Chaired by Thiow Kong Ti, NUS, Singapore)

Takeshi Sano, from the National Cancer Center Hospital, Tokyo, delivered a very concise yet comprehensive review of the epidemiology, classification, and treatment strategies for gastroesophageal junction carcinomas. He highlighted a recent randomized controlled trial from Japan, which suggests that Siewert type II tumors should be treated through an extended total gastrectomy via an abdominal approach [7]. A left thoracoabdominal approach confers no survival benefit and increases the surgical morbidity.

Live demonstration. D2 Gastrectomy (Surgeon, Takeshi Sano, National Cancer Center Hospital, Tokyo, Japan)

Many surgeons in the audience were keen to observe this live demonstration of D2 subtotal gastrectomy with
Roux-en-Y gastrojejunostomy for distal gastric cancer, performed by Takeshi Sano, an expert surgeon in gastric cancer. The surgery was filmed and transmitted live from the NUH operating theaters to the audience in the auditorium at the NUS. The participants were able to observe clearly the D2 dissection performed by Takeshi Sano. There was a lively discussion between the surgeon in the operating theater and participants in the auditorium. The live surgery took just over 2 hours, demonstrating the high skill and expertise of Takeshi Sano.

\section{Second day. November 21, 2006}

Plenary lecture 2. Chemotherapy for Advanced Gastric Cancer (Chaired by Robert Lim, NUH, Singapore)

Yoon Koo Kang, Ulsan University, Seoul, opened the second-day proceedings with a discussion that focused on chemotherapy agents for advanced gastric cancer. Continuous infusion of 5-fluorouracil (5-FU) plus cisplatin-based combination chemotherapy has been the standard for a long time. However, oral fluoropyrimidines, such as capecitabine (and S-1) may now be replacing infusional 5-FU in terms of efficacy, safety, and convenience [8].

Symposium 3. Surgery for Gastric Cancer (Chaired by Takeshi Sano, Japan, and Wai- Keong Wong, Singapore General Hospital, Singapore)

In this symposium, five topics were presented. Sudhakar Venkatesh, from the NUH, presented on imaging techniques for preoperative staging. He focused on the important role of multislide computed tomography (CT) scans in preoperative staging. Next, Chew-Wun $\mathrm{Wu}$, from Taipei Veterans General Hospital, presented the results of a randomized controlled trial conducted at his institution, comparing D1 vs D3 lymph node dissection for the treatment of gastric cancer [9]. This remains the only clinical trial that has shown a survival benefit for extended lymphadenectomy in gastric cancer surgery. Then Han-Kwang Yang, from Seoul National University Hospital, discussed the role of sentinel node biopsy in gastric cancer surgery. Sentinel node biopsy for gastric cancer, using indocyanine green, can be performed rapidly and easily, with a high detection rate. It can predict lymph node status in T1 gastric cancer with a high degree of accuracy [10]. He also described their experience with laparoscopy-assisted distal gastrectomy for early gastric cancer, reporting an advantage for the laparoscopic approach, in terms of postoperative recovery, compared to open surgery. Kent Man Chu, from Hong Kong University Hospital, spoke about surgery for locally advanced gastric cancer [11]. He described the importance of accurate preoperative staging, the extent of resection, and the role of adjuvant chemotherapy in relation to survival benefit. He also high- 
lighted that risk-adjusted mortality is substantially lower when the surgery is performed by subspecialty interested and trained surgeons.

Symposium 4. Current and Future in Chemotherapy and Radiotherapy (Chaired by Wen-Hsin Koo, National Cancer Centre, Singapore, and Robert Lim, NUH, Singapore)

Takeshi Sano began the symposium by delivering a lecture on adjuvant and neoadjuvant therapy for gastric cancer. He reviewed the literature on multimodal therapy and discussed the Japanese experience with newer agents such as S1. Next, Hans Chung, from the NUH, talked about the role of radiotherapy in adjuvant treatment for gastric cancer [12]. He highlighted a Korean study regarding the advantages of adjuvant chemoradiation after a D2 nodal dissection. The last speaker, Kian-Fong Foo, from the National Cancer Centre Singapore, reviewed current therapeutic strategies for gastrointestinal stromal tumor (GIST). He described the role of imatinib, a highly selective tyrosine kinase inhibitor, in GIST. A phase III trial of sunitinib in imatinib-resistant/-intolerant GIST showed that sunitinib was active for GIST patients who were resistant or intolerant to imatinib, and these patients showed a significant improvement in time to progression after being treated with sunitinib.

Lunch satellite symposium. The Role of Xeloda (Capecitabine) for Gastric Cancer (Chaired by WeiPeng Yong, NUH, Singapore)

For this special symposium, Yoon Koo Kang, from Ulsan University, Seoul, discussed the role of Xeloda as an effective alternative replacing infusional 5-FU in advanced gastric cancer. He highlighted a few trials that favored Xeloda combination chemotherapy as first-line treatment in patients with advanced gastric cancer, and noted the correlation of the Xeloda combination chemotherapy to complete response, overall survival, and safety profiles compared to other conventional regimens.

Teleconference. (Chaired by Jimmy So and Davide Lomanto, NUH, Singapore)

This live teleconference session (Fig. 1) involved centers from four different countries: Korea, Japan, Taiwan, and Singapore. The first session was transmitted from Korea, where Hyung-Ho Kim (Seoul National University Bundang Hospital, Korea) spoke about the role of laparoscopy-assisted radical gastrectomy. Next, Kazuhiko Nakamura (Kyushu University Hospital, Japan) discussed ESD for the treatment of early gastric cancer. Ming-Tsan Lin (National Taiwan University Hospital) then showed us a video demonstration of his novel technique of gasless laparoscopy-assisted

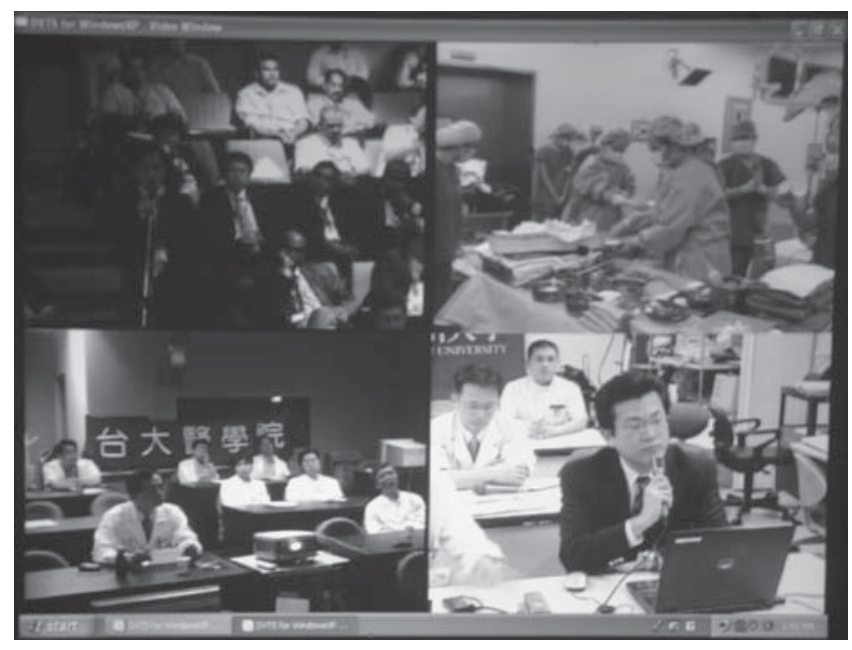

Fig. 1. Teleconference between four countries

radical gastrectomy. A lively debate ensued about the relative advantages of the different laparoscopyassisted techniques.

Live demonstration A. Laparoscopic Gastrectomy: Live Surgery from Korea (Chaired by Simon Wong, Pamela Youde, Nethersole Eastern Hospital, Hong Kong, Hong Kong, and Khong-Hee Lim, Tan Tock Seng Hospital, Singapore, and Hock-Soo Ong, Singapore General Hospital, Singapore)

In this live session from Korea, Hyung-Ho Kim demonstrated his technique for laparoscopy-assisted radical gastrectomy. The audience was impressed by his skill in performing this technically challenging procedure.

Live demonstration B. Endoscopic Submucosal Dissection (ESD) and Endoscopic Ultrasound (EUS) (Chaired by Lawrence Ho and Christopher Khor, NUH, Singapore)

This was a live session demonstrating the ESD technique for early gastric cancer. Two patients with early gastric cancer had ESD performed by Ichiro Oda, from the National Cancer Centre, Tokyo, and EUS was performed in three patients by Kenjiro Yasuda, Japan, and John Meenan, United Kingdom.

\section{Third Day, November 22, 2006}

Three workshops were held concurrently, and each workshop was conducted by distinguished overseas faculty together with local experts from Singapore to share their experiences and discuss current issues. Participants had the opportunity to practice new techniques in hands-on sessions during these workshops. 


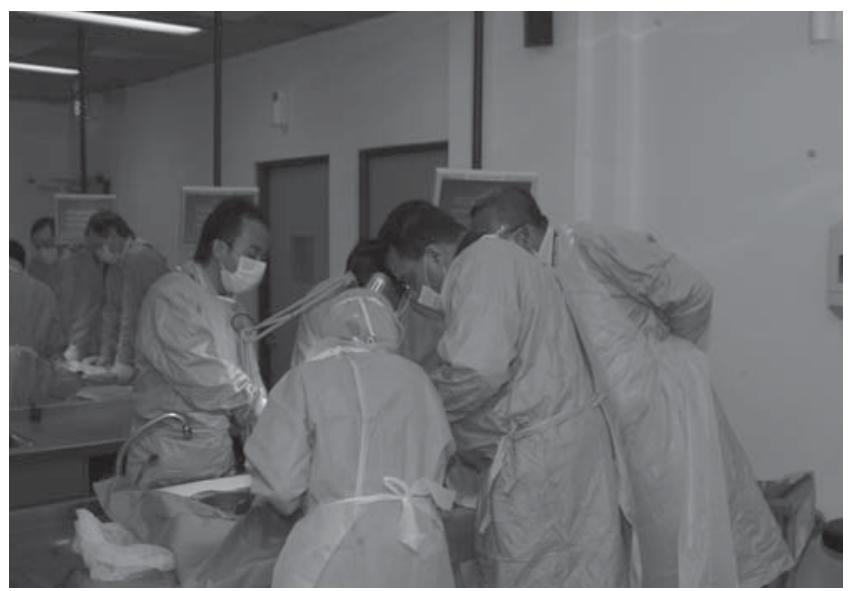

Fig. 2. Participants at the hands-on cadaveric dissection

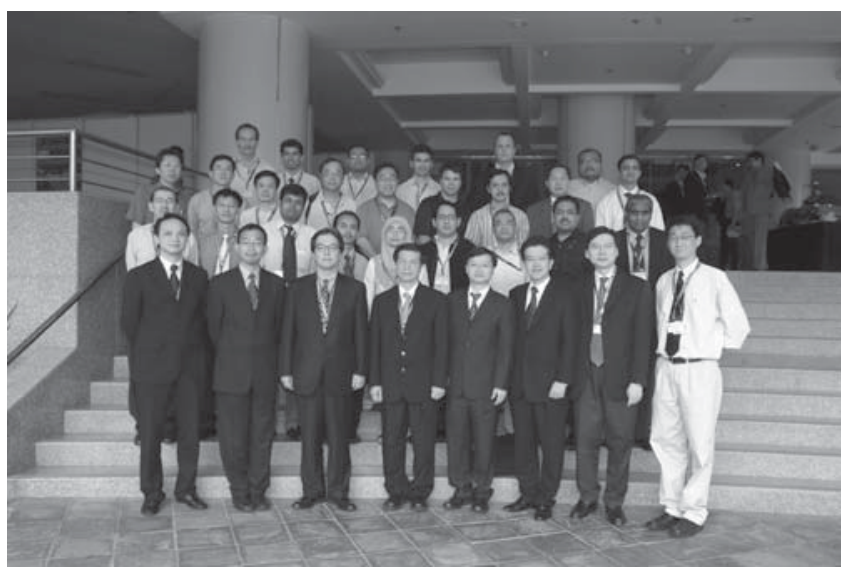

Fig. 3. Faculty and participants after the D2 gastrectomy hands-on workshop

Workshop A. D2 Gastrectomy: Hands-on Cadaveric Dissection Workshop (Course directors, Eric Gan and Jimmy So, NUH, Singapore)

The workshop started with a series of lectures and video demonstrations in the morning. Kent-Man Chu started off with his lecture on the surgical anatomy of the stomach. This was followed by a lecture from Takeshi Sano, who presented on the current Japanese classification of gastric cancer and contrasted it with other staging systems [13]. The third speaker, Chew-Wun $\mathrm{Wu}$, was very persuasive with his lecture entitled "Why do D2 gastrectomy?". He presented data from his randomized controlled trial comparing D1 vs D3 gastrectomy. Han-Kwang Yang and Takeshi Sano both showed us videos demonstrating the D2 gastrectomy technique. Participants then proceeded to the hands-on cadaveric dissection session (Fig. 2). A faculty member was assigned to each group, overseeing the cadaver as the moderator. Participants (Fig. 3) were given the opportunity to perform subtotal gastrectomy and D2 lymph node dissection with step-by-step instructions.

Workshop B. Endoscopic Submucosal Dissection (ESD) for Early Gastric Cancer: Hands-on Workshop (Course directors, Christopher Khor, Lawrence Ho, and Jimmy So, NUH, Singapore)

The program began with a short introduction and lecture on the techniques of ESD by Ichiro Oda. Then participants were given the opportunity to perform ESD on a live pig model. Next, Ichiro Oda showed a video on managing difficult situations in ESD. Philip Chiu discussed the management of complications and post-ESD care.

\section{Workshop C. ASEAN Hands-on Basic EUS Workshop}

This was held in conjunction with the Annual ASEAN EUS Forum 2006. The workshop, which consisted of lectures, video demonstrations, and a hands-on session with live animals, covered both the diagnostic and therapeutic uses of EUS.

\section{Conclusion}

The first Asia-Pacific Gastric Cancer Conference ended successfully on November 23, 2006. We feel that this conference provided an excellent opportunity in this region for physicians and research scientists to learn from one another and perhaps to collaborate on future research projects. We hope to meet our friends and colleagues at future Asia-Pacific Gastric Cancer Conferences.

\section{References}

1. Li QL, Ito K, Sakakura C, Fukamachi H, Inoue K, Chi XZ, et al. Causal relationship between the loss of RUNX3 expression and gastric cancer. Cell 2002;109:113-24.

2. Wong BCY, Lam SK, Wong WM, Ho J, Ching CK, Chen JS, et al. Helicobacter pylori eradication to prevent gastric cancer in a high-risk region of China: a randomized controlled trial. JAMA 2004;291:187-94.

3. Sung JJY, Lin SR, Ching YL, Zhou LY, To KF, Wang RT, et al. Atrophy and intestinal metaplasia 1 year after cure of $H$. pylori infection: a prospective, randomised study. Gastroenterology 2000;119:7-14.

4. Aggarwal A, Guo DL, Hoshida Y, Yuen ST, Chu KM, So S, et al. Topological and functional discovery in a gene coexpression meta-network of gastric cancer. Cancer Res 2006;66: 232-41.

5. Chiu PW, Inoue H, Satodate H, Kazawa T, Yoshida T, Sakashita M, Kudo SE. Validation of the quality of histological images obtained of fresh and formalin-fixed specimens of esophageal and gastric mucosa by laser-scanning confocal microscopy. Endoscopy 2006;38:236-40. 
6. Oda I, Saiton D, Tada M, Iishi H, Tanabe S, Oyama, et al. A multicenter retrospective study of endoscopic resection for early gastric cancer. Gastric Cancer. 2006;9:262-70.

7. Sasako M, Sano T, Yamamoto S, Sairenji M, Arai K, Kinoshita T. Left thoracoabdominal approach versus abdominal-transhiatal approach for cardia or subcardia cancer: a randomized controlled trial. Lancet Oncol 2006;7:644-51.

8. Kang HJ, Chang HM, Kim TW, Ryu MH, Sohn HJ, Yook JH, et al. Phase II study of capecitabine and cisplatin as first-line combination therapy in patients with gastric cancer recurrent after fluoropyrimidine-based adjuvant chemotherapy. Br J Cancer 2005;92:246-51.

9. Wu CW, Hsiung CA, Lo SS, Hsieh MC, Chen JH, Li AF, et al. Nodal dissection for patients with gastric cancer: a randomised controlled trial. Lancet Oncol 2006;7:309-15.
10. Park DJ, Lee HJ, Lee HS, Kim WH, Kim HH, Lee KU, et al. Sentinel node biopsy for cT1 and cT2a gastric cancer. Eur J Surg Oncol 2006;32:48-54.

11. Branicki FJ, Chu KM. Gastric cancer in Asia: progress and controversies in surgical management. Aust N Z J Surg 1998;68: 172-9.

12. Chung HT, Shakespeare TP, Wynne CJ, Lu JJ, Mukherjee RK, Back MF. Evaluation of radiotherapy protocol based on INT0116 for completely resected gastric adenocarcinoma. Int J Radiat Oncol Biol Phys 2004;59:1446-53.

13. Japanese Gastric Cancer Association. Japanese classification of gastric carcinoma. 2nd English edition. Gastric Cancer 1998;1:1024. 